\title{
Effect of Microfocused Ultrasound on Facial Rejuvenation: Clinical and Histological Evaluation
}

\author{
${ }^{1}$ Federal University of Rio Grande do Norte (UFRN), Natal, Brazil \\ ${ }^{2}$ University Centre of Rio Grande do Norte (UNI-RN), Natal, Brazil \\ ${ }^{3}$ Michelle Meleck Advanced Aesthetics, São Paulo, Brazil \\ ${ }^{4}$ Estácio de Sá University, Rio de Janeiro, Brazil \\ ${ }^{5}$ Universidad Finis Terrae, Santiago, Chile \\ ${ }^{6}$ Potiguar University (UNP), Natal, Brazil \\ ${ }^{7}$ Campinas State University (UNICAMP), Campinas, Brazil \\ ${ }^{8}$ Fellow da West Virginia University, Morgantown, USA \\ Email: rodrigomarcelvalentim@gmail.com
}

Patricia Froes Meyer ${ }^{1,2}$, Michelle Meleck ${ }^{3}$, Fabio dos Santos Borges ${ }^{4}$, Esteban Fortuny5, Stephany Luanna Queiroga Farias ${ }^{6}$, Felipe Alexander Caldas Afonso ${ }^{1}$, Ciro Dantas Soares 7 , Eneida de Morais Carreiro ${ }^{2}$, Rodrigo Marcel Valentim da Silva ${ }^{1}$, André Luiz Miranda Barbosa ${ }^{8}$

How to cite this paper: Meyer, P.F., Meleck, M., dos Santos Borges, F., Fortuny, E., Farias, S.L.Q., Afonso, F.A.C., Soares, C.D. de Morais Carreiro, E., da Silva, R.M.V. and Barbosa, A.L.M. (2021) Effect of Microfocused Ultrasound on Facial Rejuvenation: Clinical and Histological Evaluation. Journal of Biosciences and Medicines, 9, 112-125.

https://doi.org/10.4236/jbm.2021.97012

Received: May 4, 2021

Accepted: July 12, 2021

Published: July 15, 2021

Copyright $\odot 2021$ by author(s) and Scientific Research Publishing Inc. This work is licensed under the Creative Commons Attribution International License (CC BY 4.0).

http://creativecommons.org/licenses/by/4.0/ (c) (i) Open Access

\begin{abstract}
Introduction: Micro focused ultrasound (MFU) is formed by mechanical waves emitted in a focused manner, concentrating thermal energy on a certain point or region, promoting a clinical improvement in wrinkles. Objective: evaluate the effects of micro focused ultrasound on facial rejuvenation. Methodology: This is an experimental study. The sample consisted of 30 volunteers, who were evaluated before treatment, 45 and 90 days after its beginning. A single application of the MFU was performed across the facial region. The parameters used were: $10 \mathrm{MHz}$ cartridge with depth of $1.5 \mathrm{~mm}, 7 \mathrm{MHz}$ with depth of $3 \mathrm{~mm}$, and $4 \mathrm{MHz}$ with focal depth of $4.5 \mathrm{~mm}$; doses ranged from 0.1 to $2.0 \mathrm{~J}$, according to the sensitivity of the volunteers treated, and the application time was of approximately 90 minutes. Clinical photographic analysis and facial symmetry analysis using Dolphin Imaging software were performed, and validated questionnaires. Two volunteers were submitted to blepharoplasty surgery, and one to rhytidoplasty surgery 45 days after the application of the MFU. Samples of the treated skin were taken after the surgical procedures and submitted to histological and immunohistochemical analyses. The volunteers answered a questionnaire to assess adverse reactions and satisfaction. Results: We observed a visible clinical improvement on the images by photography, and an improvement of facial symmetry in the measure-
\end{abstract}


ments of the paralateronasal projection $(\mathrm{p}=0.007)$, left lateral mandibular $(\mathrm{p}$ $=0.01)$, and right lateral mandibular $(\mathrm{p}=0.008)$. The histological analysis showed an increase in the amount of fibroblasts $(\mathrm{p}=0.02)$, blood vessels $(\mathrm{p}=$ $0.0062)$ and inflammatory cells $(\mathrm{p}=0.0036)$, in addition to the production of type 1 collagen. In the immunohistochemical analysis, we observed the presence of the macrophage marker IHQ: CD68, indicating phagocytosis of adipose tissue and the presence of fibrosis. In the analysis of adverse reactions, only transient hyperemia was observed with the presence of pain during application. Additionally, the volunteers expressed high level of satisfaction (93\%) with the treatment Conclusion: The MFU promoted the production of collagen, reorganization of the dermis and, clinically, a reduction of sagging and wrinkles, with increased facial symmetry.

\section{Keywords}

Skin Aging, Mechanical Waves, Sagging, Wrinkles

\section{Introduction}

Micro focused ultrasound (MFU) is a technology used to treat sagging skin, especially on the different regions of the face and neck. In recent years, the effectiveness and safety of this device have been well documented [1] [2]. The physical mechanism involved consists of the transmission of mechanical waves, which are converted into concentrated thermal energy at the points of application, inducing a controlled and localized inflammatory response. It is a non-ablative and non-invasive response, promoting an increase in the production of collagen and elastin [3].

MFU can present frequency variation of 1 to $10 \mathrm{MHz}$ and controlled depth of the focal point, through specific "cartridges" containing appropriate transducers for the controlled emission of focused sound waves. The action of the MFU with the concentration of energy at the focal point promotes a thermal response, without damage to blood vessels, peripheral sensory nerves, and adjacent tissues. Thus, the focused sound waves promote lifting in the superficial and deep layers of the skin, caused by the production of collagen fibers, and the sound waves can also reach the muscles of the region [1] [3] [4].

There has been an increase in the use of this resource in clinical practice for treatment of flaccidity and wrinkles in the facial region, despite the guarantee of safety in the correct use of the technique, the biological response is well founded, however, the clinical improvement with facial rejuvenation and the adequacy of symmetry still need further study.

Despite the guarantee of safety in the correct use of the technique, the biological response is well founded, however, a larger amount of clinical studies is needed, a more solid basis, as well as histological and immunohistochemical analyses. In this context, this study aims to investigate the effects of MFU on fa- 
cial rejuvenation, analyzing clinical changes in facial symmetry, the level of satisfaction and the manifestation of possible adverse responses, as well as the visualization of the quantity and type of collagen. and elastic fibers after MFU application.

\section{Methodology}

The volunteers were seen at Clinical Eterna and Clinical Michele Meleck outpatient clinics, located respectively in the cities of Natal/RN and São Paulo/SP. This study was submitted to the Research Ethics Committee (CEP) of Universidad Potiguar-UnP, having received approval under number 3.726.965. The participants were instructed on the procedures to be performed and signed the Free and Informed Consent Form.

\subsection{Population and Sample}

The sample included in this study was composed of 30 women, aged 30 to 60 years old, who presented facial skin aging (tissue flaccidity, wrinkles, elastosis), did not have healing problems, keloids or collagen-related disorders, and did not have any serious metabolic diseases.

The justification for an exclusive selection of women was to standardize the sample, in relation to hormonal characteristics of gender, skin aspects, among others. The use of a sample of both sexes could generate a variation in the analysis mechanism.

Volunteers who gave up treatment before the end of the interventions, were absent from evaluations or treatment sessions, and who had any complication after the application were excluded from the study. The sample was divided into 3 groups of 10 volunteers, according to their age group, 30 to 40 years old, 41 to 50 years old, and 51 to 60 years old. Two research volunteers underwent unilateral facial treatment and subsequently underwent blepharoplasty surgery, and one research volunteer underwent unilateral facial treatment, and subsequently underwent rhytidoplasty surgery.

\subsection{Research Tools}

The instruments used to collect data for this research were the Facial Assessment Protocol (PAF), validated by Micussi et al. (2008) [5], in which the following topics were addressed: identification, anamnesis, physical examination, analysis of wrinkles and sagging of skin, phototype classification (Fitzpatrick), and Tsuji classification.

For the analysis of facial symmetry, we used a photographic analysis system in 2D and 3D, with a software called Dolphin Imaging Brazil-Renovatio, using parallel lines and angulations to analyze facial symmetry, configuring a standardization of the facial image for the analysis of tissue flaccidity [6] [7].

We used patient satisfaction analysis adapted questionnaires, Segot-chicq et al. (2007) [8] and the Global Aesthetic Improvement Scale-GAIS, by Narins 
(2003) [9], which is used to classify response to treatments, allowing for a comparative assessment at different times after the therapeutic intervention.

For the photographic record, a NikonD500 ${ }^{\mathrm{TM}}$ camera was used. The treatment was carried out using micro focused ultrasound equipment, model Heros Hi$\mathrm{fu}^{\mathrm{TM}}$, manufactured by Fismatek ${ }^{\mathrm{TM}}$.

\section{Procedures}

After the selection, the participants were informed about the procedures to be performed and, those who were in agreement signed the free and informed consent form. Next, they were submitted to evaluation using the Facial Evaluation Protocol (PAF). The photos were registered with the volunteer in an orthostatic position, where images were collected in the anterior and lateral views (right and left). The camera used was the same in all photos. It was placed on a support tripod at a height of $66 \mathrm{~cm}$ from the floor and at a distance of $55 \mathrm{~cm}$ from the participant, for better viewing and standardization of the photos. At the end, the photos were evaluated using an analysis software (Dolphin Imaging version 12.0) to assess the results.

The therapeutic procedures were performed with the Heros HIFU ${ }^{\mathrm{TM}}$ micro focused ultrasound equipment, manufactured by Fismatek ${ }^{\mathrm{TM}}$ in a single session. For the treatment session, all volunteers were placed in the supine position and the applicator was positioned on the facial region. The parameters used were: 10 $\mathrm{MHz}$ cartridge with depth of $1.5 \mathrm{~mm}, 7 \mathrm{MHz}$ with depth of $3 \mathrm{~mm}$, and $4 \mathrm{MHz}$ with focal depth of $4.5 \mathrm{~mm}$; doses ranged from 0.1 to $2.0 \mathrm{~J}$, according to the sensitivity of the volunteers treated, and the application time was of approximately 90 minutes.

The treatment area comprised the entire facial region (Full Face), specifically covering the frontal region, lateral commissure of the lower and upper eyelids, buccinator and masseter muscle region, upper and lower lip, mandible, nasogenian groove, and orbicularis oris. To perform the procedure, the region was cleaned with antiseptic soap. Then, the facial region was divided into sub-areas and marked with a white pencil for greater precision of the micro focused ultrasound, which was applied by positioning the cartridge with minimal manual pressure on the skin, making a pass with each cartridge $(1.5 \mathrm{~mm}, 3 \mathrm{~mm}, 4.5 \mathrm{~mm})$ over each area marked for treatment. For the coupling of the cartridge to the skin, a common gel was used.

The pulses were fired following the lines. The distance between one line and another was approximately $3.0 \mathrm{~mm}$, completing the marked "column", without overlapping shots. The frontal, orbicular, zygomatic and mandibular regions were treated with the $4.5 \mathrm{~mm}$ cartridge (total of 154 lines of shots). The frontal regions, eye commissure, lower eyelid, zygomatic, mandible, and nasogenian groove (total of 152 shooting lines) were treated using the $3 \mathrm{~mm}$ cartridge. The frontal, orbicular region of the eyes, lower and upper eyelids, zygomatic, mandibular, and nasogenian groove (total of 310 lines) were treated with the $1.5 \mathrm{~mm}$ cartridge. 
During the evaluations, the volunteers were submitted to the protocol, having their photographs taken, and facial symmetry analyzed. The 2D photographic analysis was done using the Dolphin Imaging Brazil-Renovation software and the regions analyzed were: the nasolabial crease-frontal view, formed by the angle of two tangent lines between the labial commissure and nasolabial crease, where its increase means the crease was repositioned to the medial region (decreased "groove"); and the L cervical measurement-cervical angle-lateral view, which is formed by the angle between two lines tangent to the upper and lower cervical projections, so that its increase indicates a decrease in sagging [6] [7].

In the evaluation of facial symmetry, we were able to observe the following measurements: the paralateronasal projection, which consists of the angle formed by two lines tangent to the lateral nasal and nasolabial projections that indicated an increase in the paralateronasal projection (characterizing the filling in the nasal region), in which case the reduction indicates a decrease in sagging; the angle of the lateral mandibular projection-frontal view, which consists of the angle formed by two tangent lines formed by the lateral and inferior projections of the zygomatic region, so that its increase indicates a reduction of the projection of the lateral palpebral wrinkle-frontal view, formed by the angle between the two tangent lines between the ocular corners and the lateral crease, which means that the crease has been repositioned superiorly (the flaccidity has decreased); and, finally, the eyelid crease-side view, with the angle formed by two tangent lines being the lateral palpebral crease and the back of the nose, and the increase means that the crease was elevated (sagging has decreased) [6] [7].

At the end of the treatment, three volunteers underwent a blepharoplasty and rhytidoplasty surgical procedure, and during the surgery, a skin biopsy technique was performed on the upper eyelids and on the face to collect material. The skin fragments removed were sent for analysis with optical and microfocal microscopy, to identify the amount and type of collagen, as well as the elastic fibers, through histological and immunohistochemical analysis. The volunteers were sedated and, for the biopsy and before the rhytidoplasty, incisions were made in the upper eyelid margin and regions of the lateral and lower face, with removal of skin flap and adipose tissue. An absorbable Vicryl 3-0 suture was used to finish the procedure.

The flaps of the eyelid and face removed during the surgery were embedded in paraffin for the preparation of the slides and qualitative analysis by means of optical microscopy. The evaluations were conducted by means of hematoxylin and eosin (HE) stain 40x, 100x, 400x, to verify the morphology of the dermal, epidermal, and hypodermic tissues. The criteria analyzed were the presence of edema, acute and chronic inflammatory process, presence of fibroblasts, neo collagen and mature collagen. The immunohistochemical criteria analyzed were the presence of CD68 macrophage, the identification of necrosis, adipolysis process, and type of collagen.

Each volunteer answered a satisfaction and sensation questionnaire at the end 
of the last treatment session [8] [9]. The reassessment was done three times, at the initial moment, 45 and 90 days after the single session, with the repetition of all the procedures and photos mentioned. The volunteers were attended to with the treatment adequately structured for the study proposed, in a location containing an appropriate environmental cooling system and good hygiene and lighting conditions.

\section{Data Analysis}

Descriptive and inferential statistics of the data were performed using the SPSS 22.0 program (Statistical Package for the Social Science-version 22.0). The normality of the data was observed by the Kolmogorov-Smirnov (KS) test. For comparisons of the measurements obtained in all evaluations (initial, 30, 60, and 90 days) the ANOVA test of repeated measures, with post hoc Bonferroni was used. The $95 \%$ significance level was adopted $(\mathrm{p}<0.05)$.

\section{Results}

The study volunteers had white skin, with phototypes from I to III, with the presence of static and dynamic wrinkles. According to the analysis of the PAF evaluation protocol, most had mixed skin, with Tsuji classification of superficial aging in most of the group and according to Lapiere and Pierard, and with epidermal thinning in $72 \%$ of the group. About $71 \%$ of the group does not use sunscreen and, those who do, apply it twice a day on average.

\subsection{Clinical Analysis}

Figures 1(a)-(b) show the photographic analysis of patients who underwent blepharoplasty surgery, before the procedure, with indication of the changes caused by the unilateral application of the MFU.

\subsection{Analysis of Facial Symmetry}

Table 1 represents the results of the analysis of the facial symmetry software.

In the facial symmetry evaluation, it is possible to observe that there was a significant reduction of the L Paralateronasal Projection measure after 90 days of the initial intervention, $(p=0.007)$ indicating an increase in the projection (filling) of the medial region of the face. We can see that the measures of F-Lateral Mandibular Projection R ( $p=0.01)$, F-Lateral Mandibular Projection L (0.008). In the analysis of the right and left F-Nasolabial Wrinkle, a significant increase was observed in both groups $(\mathrm{p}=0.651)$ and $(\mathrm{p}=0.71)$. In the analysis of the right and left F-Lateral Eyelid Wrinkles, we did not observe significant increase $(\mathrm{p}=0.61$ and $\mathrm{p}=0.74)$.

\subsection{Qualitative and Quantitative Analysis of the Histological Material of the Skin Fragment}

Figure 2 and Figure 3 show the results of the histological analysis of the material removed in the blepharoplasty, in which the following were analyzed: inflammatory process, presence of collagen, and types of collagen produced. 
Table 1. Analysis of facial symmetry with a comparison of means and standard deviation of variables.

\begin{tabular}{cccc}
\hline & Initial & Final & P value \\
\hline L-Paralateronasal Projection & $147.94 \pm 6.11$ & $142.97 \pm 12.05$ & $0.007^{\star}$ \\
F-Lateral Eyelid Wrinkle L & $45.72 \pm 4.13$ & $53.26 \pm 5.34$ & 0.69 \\
F-Lateral Eyelid Wrinkle R & $46.26 \pm 4.54$ & $52.33 \pm 7.79$ & 0.53 \\
F-Lateral Mandibular Projection L & $124.31 \pm 11.73$ & $133.31 \pm 7.87$ & $0.01^{\star}$ \\
F-Lateral Mandibular Projection R & $122.54 \pm 14.47$ & $133.31 \pm 7.87$ & $0.008^{\star}$ \\
F-Nasolabial Wrinkle L & $137.54 \pm 11.04$ & $137.13 \pm 9.56$ & 0.65 \\
F-Nasolabial Wrinkle R & $130.42 \pm 9.14$ & $136.03 \pm 9.64$ & 0.71 \\
\hline
\end{tabular}

${ }^{\star}$ Significant statistical difference.
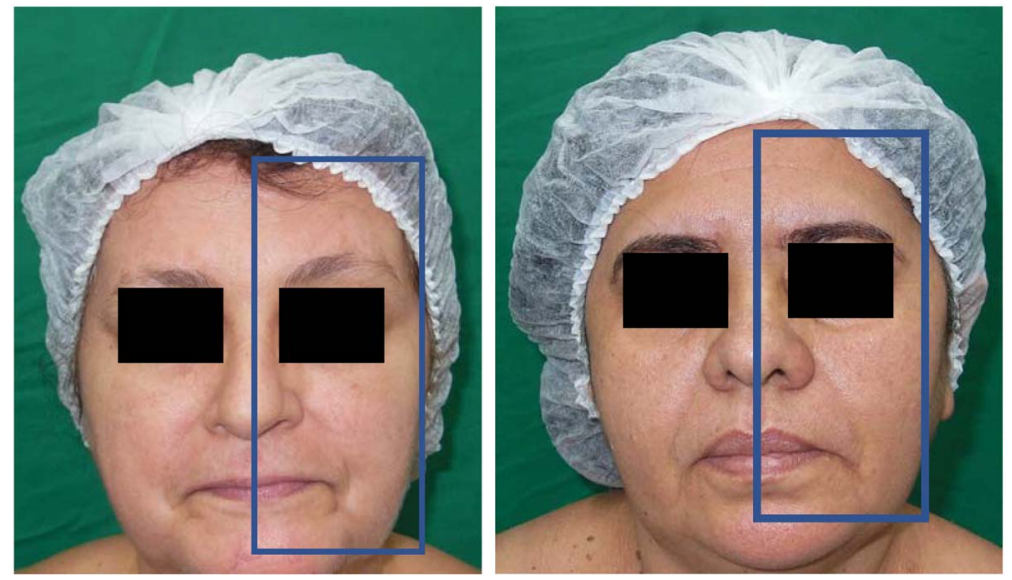

(a)
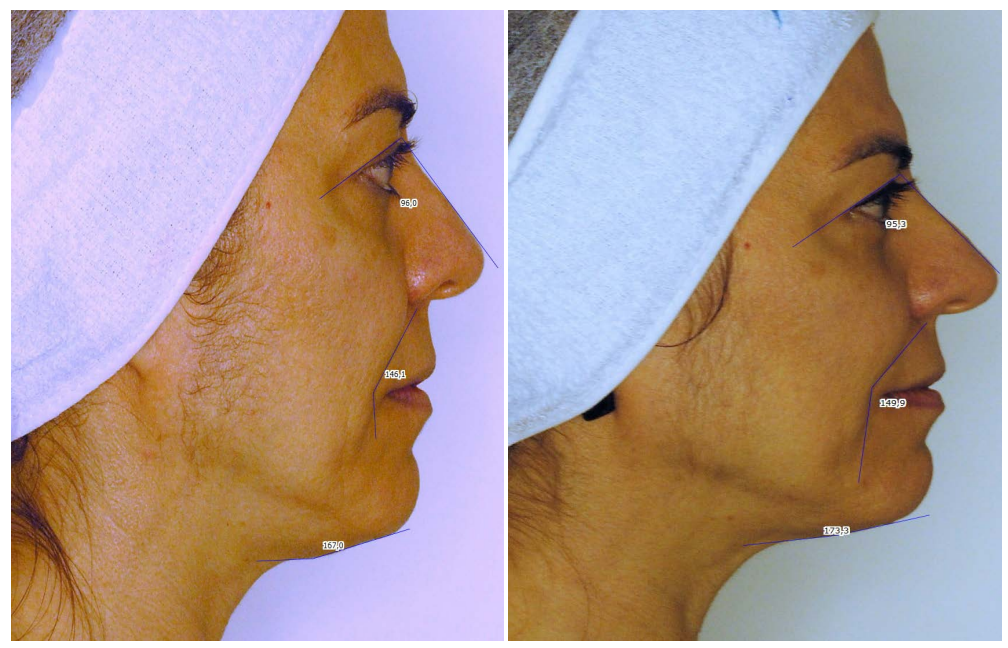

(b)

Figure 1. (a) A: Clinical analysis of the photos. B: Patient submitted to unilateral application of the MFU, which was performed on the left side. B: Patient submitted to unilateral application of the MFU, which was performed on the left side. Note the asymmetry between the two sides, with a noticeable lifting effect on the left side. (b) Analysis of facial symmetry software regarding the angles before and after treatment. 


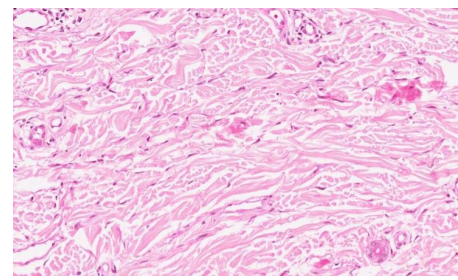

(a)

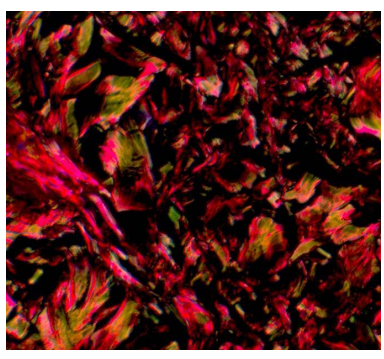

(c)

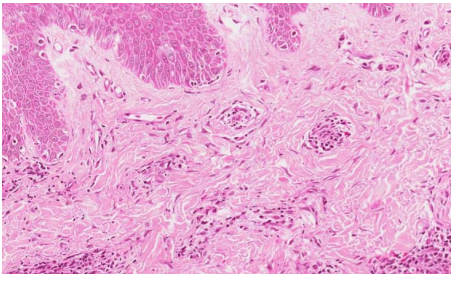

(b)

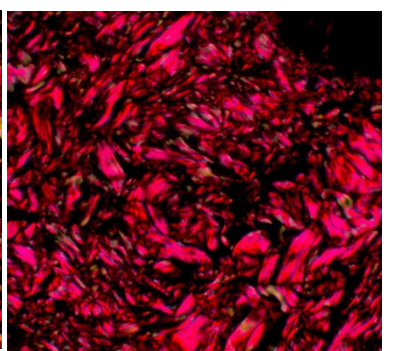

(d)

Figure 2. Histological analysis (a) Control group: dermis with a normal number of fibroblasts, vessels, and inflammatory cells. (b) Treated group: an evident increase in the number and size of fibroblasts, a larger number of vessels and inflammatory cells than in the Control group. (c) Control group with a predominance of type III collagen (green). (d) Treated group with evident increase of type I collagen (orange), when compared to type III collagen (green).

Fibroblasts

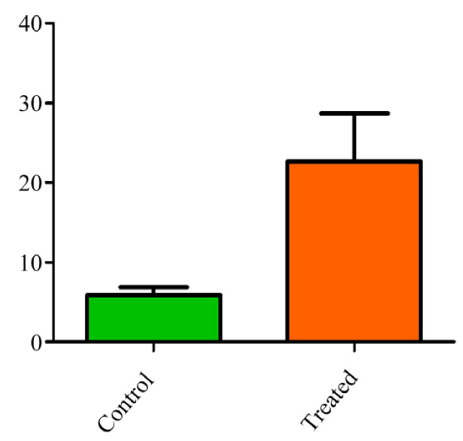

(a)
Vessels

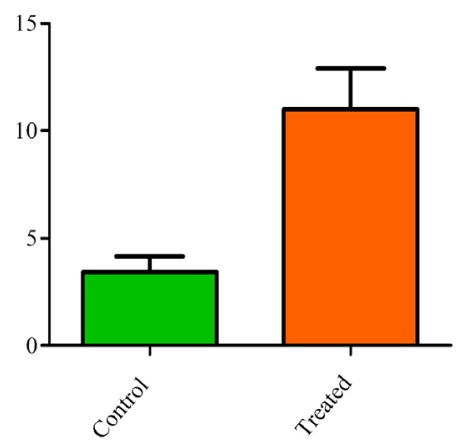

(b)

Inflammatory cells

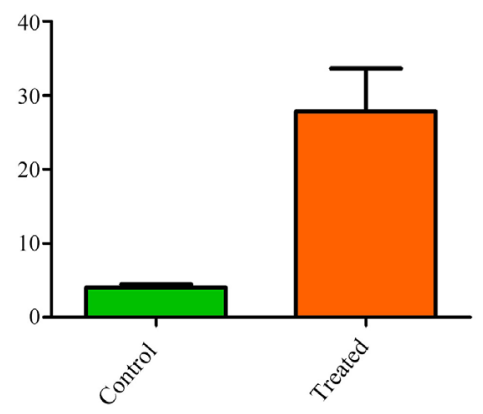

(c)

Figure 3. Comparative analysis of the number of cells between the Control and Treated groups. (a) Number of fibroblasts. (b) Number of blood vessels. (c) Number of inflammatory cells. 
Figure 2(a) and Figure 2(b) show the qualitative results of the dermis on the side without or with the application of the MFU. Figure 2(c) and Figure 2(d) show the analysis of the predominant type of collagen in the tissue. Figures 3(a)-(c) show the inferential analyzes between the control and treated sides, observing differences between the control side compared to the treated side in the assessment of number of fibroblasts $(p=0.02)$, blood vessels $(p=0.0062)$ and inflammatory cells $(\mathrm{p}=0.003)$.

Figure 4(a) shows the results of the immunohistochemical analysis, showing a small amount of CD68 macrophages in the control group. Figure 4(b) corresponds to the group treated with the presence of necrosis and fibrosis in dermal and hypodermic tissue. The presence of macrophages indicates a process of phagocytosis of hypodermic necrotic tissue and the presence of fibrosis.

In Figure 4(c), control group, we verified the presence of macrophages in the adipose tissue, and in Figure 4(d), treated group, the presence of macrophages positive for adipophilin, indicating the process of adipolysis and lipolysis. Figure 4(e) shows the adipose tissue in the control group, and $4 \mathrm{~F}$ and $4 \mathrm{G}$ characterize the region of the eyelid and face, indicating the presence of areas of coagulation and fibrosis, which are more intense in the region of the face skin. Coagulation characterizes the maintenance of a chronic inflammatory process, related to fibroblast proliferation, associated with greater collagen formation in the long term.

\subsection{Results of the Adverse Reaction Questionnaires}

Regarding adverse reactions after application, we observed that $55 \%$ of the volunteers reported hyperemia, however, $70 \%$ of this sample reported that this symptom disappeared after application. Regarding the texture of the skin, $46 \%$ of the volunteers reported that their skin was firmer. About $93 \%$ of the volunteers reported the presence of pain, from mild to severe, during the application, and $43 \%$ reported decreased sensitivity after the application. However, another $43 \%$ reported local hypersensitivity. No shocks, trauma, edema, ecchymosis, bruising, and burns were observed after the MFU applications.

\subsection{Results of the Satisfaction Questionnaires}

It was a questionnaire with individual questions reported by volunteers, who were asked about satisfaction with the treatment. The answers were freely given by the volunteers, so one idea would be to identify a self-perception about the results. It was observed that the volunteers believed to have a satisfactory result $93 \%$ and $7 \%$ found the result unsatisfactory, with $47 \%$ reporting that MFU is an excellent treatment, and $40 \%$ classifying it as a good treatment. In the global improvement scale analysis, $50 \%$ reported improvement, $36 \%$ said they were much better. And 14\% described the result as good.

\section{Discussion}

The purpose of this study was to analyze the effects of MFU on facial aging, with 
the clinical purpose of reducing sagging and wrinkles. The reduction of collagen characterized in wrinkles promotes a disorganization of connective tissue fibers [10].

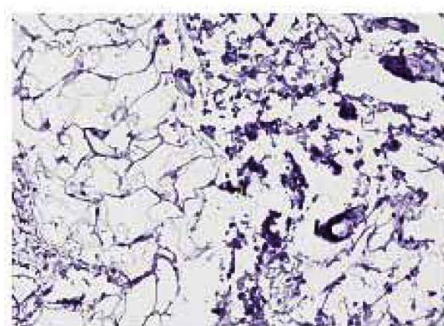

(a)

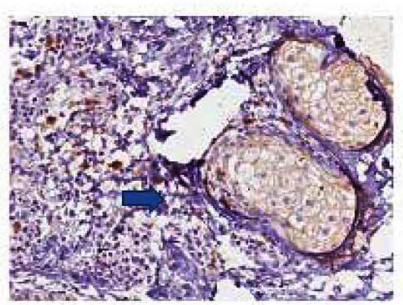

(c)

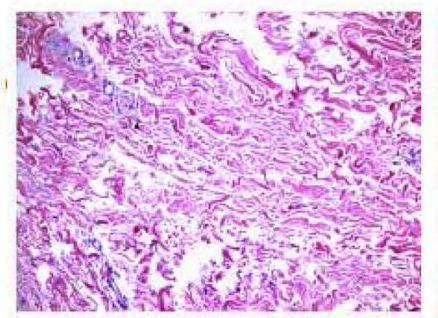

(e)

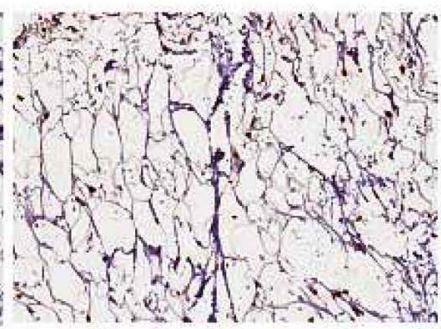

(b)

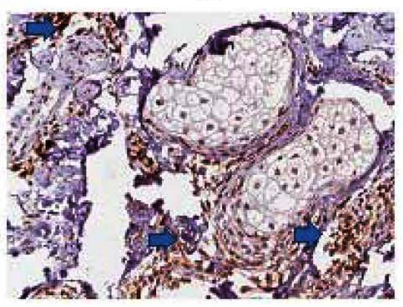

(d)

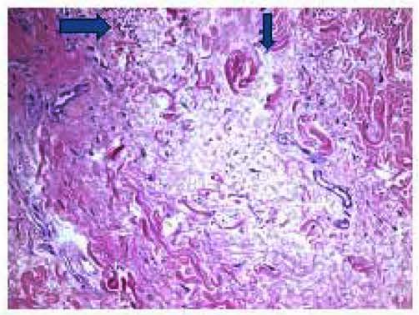

(f)

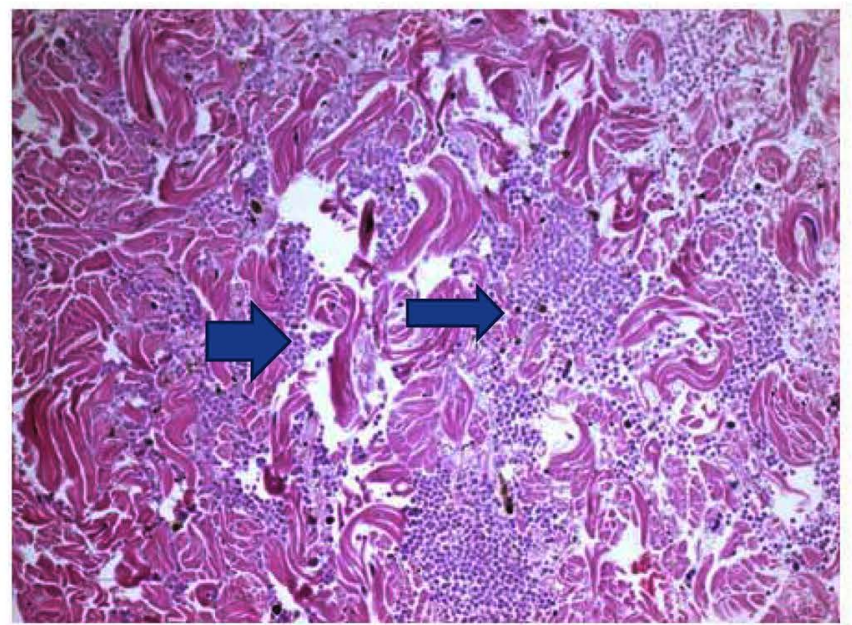

(g)

Figure 4. (a) Control: Normal adipose tissue, with few CD68 macrophages. (b) Treated: Presence of CD68 macrophages: necrosis and fibrosis dermal and hypodermic tissue. (c) Control: Normal adipose tissue, with few macrophages phagocyting adipose tissue. (d) Treated: Presence of macrophages positive for adipophilin adipolysis/lipolysis process. (e) Control. (f) Treated: Connective tissue of the eyelid region (coagulation and fibrosis area). (g) Treated: Connective tissue of the face region (coagulation and fibrosis area). 
The analysis of the photos showed a change in the cutaneous tissue, more pronounced in the mentonian region and neck. This clinical response of reduced flaccidity and wrinkles has been the alternative of several patients in clinical practice to promote this type of change. The use of MFU has been shown to be effective for this type of response in other studies [11] [12], which also made use of photographs or clinical analysis methods, featuring a reduction of flaccidity [3] [11] [12].

It can be observed that the evaluation of the photographs using the facial symmetry analysis software, by the definition of sagging and wrinkles, alterations were found at the paralateronasal and mandibular levels. The reduction in the paralateronasal projection indicates an increase in the projection (filling) of the medial region of the face. As for the mandibular region, there is a significant increase in the cervical line that decreased the projection of the mentonian region and neck (less sagging in the region). Other systems based on photos and angle measurements for symmetry analysis have also analyzed responses similar to those investigated in these studies, showing results that indicate improved facial alignment and reduction of flaccidity after the application of the MFU [13].

In the histological analysis, we observed increased fibrosis in the face region compared to the eyelid region. And these results were observed 45 days after the intervention, while most studies do a later analysis, 90 days after the intervention. The probable reason is the greater sensitivity in the eyelids during the application of the MFU, which limits the use of higher doses. Therefore, in view of the results found, we have observed a tissue response to the thermal action of the procedure, generating points of fibrous lesion and probable plan adhesion, generating the lifting effect seen in clinical practice. This response is also characterized by the penetration of mechanical waves in the deep region of the dermis, without affecting more superficial tissues, so this is an indicative factor for the localized collagen modulation response [14].

The immunohistochemical analysis also demonstrates the effects of the MFU, confirming the responses of scientific literature regarding this technology, being that the production of thermal action points will generate fibroblast activation, collagen production, and fibrosis formation. This response on biological tissue has already been observed by different mechanisms of transmission of mechanical or ultrasonic waves, being the case of the MFU, also a modality with thermal effect that intensifies this response. In this study, we noticed that the thermal modulation generated by the MFU resulted in an increased number of fibroblasts, an increase in collagen, with a predominance of type 1 collagen, and an increase in the number of inflammatory cells and blood vessels. The combination of these mechanisms is responsible for the organization of the tissue at immunohistochemical and histological level, which orders the fibers and allows for clinical improvement in the appearance of the skin, with reduction of flaccidity and lifting effect [4] [15] [16].

Another response observed regards adipose tissue, indicating a reduction in 
adipocyte volume, showing signs of adipolysis. The presence of thermal necrosis was observed, with CD68 markers and adipophilin, confirming a high number of macrophages, which indicates a process of phagocytosis of this tissue, and destruction of the adipose tissue [16] [17].

Regarding adverse reactions, all reactions were controlled and expected such as the presence of temporary hyperemia, lasting from 1 to 3 hours after the application. This response is characteristic of the MFU technique, which promotes an increase in temperature and, consequently, the appearance of responses that induce controlled inflammation after application [18].

We have observed that $92 \%$ of the volunteers perceived greater firmness in the skin, a response already verified in other studies and related to the increase in the quantity and quality of collagen produced by fibroblasts. Clinically, tissue with unchanged epidermal function is observed, with a progressive increase in skin elasticity and histological remodeling of the dermis [19] [20].

The painful sensation is characteristic in the application of the MFU, and about $93 \%$ of the volunteers in this study have reported this adverse response. This result was already expected due to the inflammatory induction and release of irritating substances to nerve endings due to the thermal response. Additionally, unlike other studies, there was no use of anesthetic substances before the interventions [20] [21].

The literature reports other adverse responses after the application of the MFU, such as burns, ecchymosis, bruises, edema, among others [21]. However, in this study, no adverse responses like these were reported. In fact, we did not identify relevant clinical complications, only hyperemia and pain during application.

Regarding the satisfaction of the volunteers in this study, they expressed considerable satisfaction, with $47 \%$ of the volunteers classifying the micro focused ultrasound treatment as excellent, $93 \%$ of the volunteers reporting satisfaction with the treatment and saying they would recommend it to other people, and $86 \%$ noticing improvement in overall aesthetic after the treatment. Other studies have also reported patient satisfaction with the results observed after applying the MFU to facial flaccidity with the presence of wrinkles [3] [4] [16] [22].

There were limitations to this study, such as the absence of a control or placebo group to compare results, as well as limited clinical methods for assessing flaccidity. We suggest that further studies be conducted with other parameters, and with a follow-up analysis.

\section{Conclusion}

It was observed that the use of MFU promotes the production of collagen, reorganization of the dermis, and clinical improvement of flaccidity and wrinkles, with improved facial symmetry, and minimized adverse reactions, as well as a good level of satisfaction among the patient who received the intervention. Therefore, the MFU is an effective intervention in facial sagging. 


\section{Acknowledgements}

We would like to thank Clínica Eterna, Natal/RN and Clínica Michele Meleck, from São Paulo/SP for providing the structure and material for conducting the research.

\section{Conflicts of Interest}

The authors declare no conflicts of interest regarding the publication of this paper.

\section{References}

[1] Hart, D.R., Fabi, S.G., White, W.M., Fitzgerald, R. and Goldman, M.P. (2015) Conceitos Atuais no Uso de PLLA: Sinergia Clínica Notável com Uso Combinado de Ultrassom Microfocado e Ácido Poli-L-Láctico na Face, Pescoço e Decote. Plastic and Reconstructive Surgery, 136, 180S-187S.

[2] Casabona, G. and Kaye, K. (2019) Facial Skin Tightening with Microfocused Ultrasound and Dermal Fillers: Considerations for Patient Selection and Outcomes. Journal of Drugs in Dermatology, 18, 1075-1082.

[3] Wulkan, A.J., Fabi, S.G. and Green, J.B. (2016) Microfocused Ultrasound for Facial Photorejuvenation: A Review. Facial Plastic Surgery, 32, 269-275. https://doi.org/10.1055/s-0036-1584129

[4] MacGregor, J.L. and Tanzi, E.L. (2013) Microfocused Ultrasound for Skin Tightening. Seminars in Cutaneous Medicine and Surgery, 32, 18-25.

[5] Micussi, M.T.A.B.C., Oliveira, T.C.M., Meyer, P.F. and Araújo, F.R.O. (2008) Protocolo de avaliação facial: Uma proposta fisioterápica. Fisioterapia Brasil, Suplemento especial.

[6] Xia, J.J., Gateno, J. and Teichgraeber, J.F. (2009) New Clinical Protocol to Evaluate Craniomaxillofacial Deformity and Plan Surgical Correction. Journal of Oral and Maxillofacial Surgery, 67, 2093-2106. https://doi.org/10.1016/j.joms.2009.04.057

[7] Bobek, S., Farrell, B., Choi, C., Farrell, B., Weimer, K. and Tucker, M. (2015) Virtual Surgical Planning for Orthognathic Surgery Using Digital Data Transfer and an Intraoral Fiducial Marker: The Charlotte Method. Journal of Oral and Maxillofacial Surgery, 73, 1143-1158. https://doi.org/10.1016/j.joms.2014.12.008

[8] Narins, R.S., Brandt, F., Leyden, J., Lorenc, Z.P., Rubin, M. and Smith, S. (2003) A Randomized, Double-Blind, Multicenter Comparison of the Efficacy and Tolerability of Restylane versus Zyplast for the Correction of Nasolabial Folds. Dermatologic Surgery, 29, 588-595. https://doi.org/10.1046/j.1524-4725.2003.29150.x

[9] Segot-Chicq, E., Compan-Zaouati, D., Wolkenstein, P., Consoli, S., Rodary, C., Delvigne, V., Guillou, V. and Poli, F. (2007) Development and Validation of a Questionnaire to Evaluate How a Cosmetic Product for Oily Skin Is Able to Improve Well-Being in Women. The Journal of the European Academy of Dermatology and Venereology, 21, 1181-1186. https://doi.org/10.1111/j.1468-3083.2007.02193.x

[10] Friedman, Paul, M. et al. (2002) Safety Data of Injectable Non-Animal Stabilized Hyaluronic Acid Gel for Soft Tissue Augmentation. Dermatological Surgery, 28, 491-494. https://doi.org/10.1097/00042728-200206000-00010

[11] Lee, H.S., Jang, W.S., Cha, Y.J., et al. (2012) Multiple Pass Ultrasound Tightening of Skin Laxity of the Lower Face and Neck. Dermatologic Surgery, 38, 20-27. https://doi.org/10.1111/j.1524-4725.2011.02158.x 
[12] Lu, P.H., Yang, C.H. and Chang, Y.C. (2017) Quantitative Analysis of Face and Neck Skin Tightening by Microfocused Ultrasound with Visualization in Asians. Dermatologic Surgery, 43, 1332-1338. https://doi.org/10.1097/DSS.0000000000001181

[13] Oni, G., Hoxworth, R., Teotia, S., Brown, S. and Kenkel, J.M. (2014) Evaluation of a Microfocused Ultrasound System to Improve Sagging and Tightening of the Skin on the Underside. Aesthetic Surgery Journal, 34, 1099-1110. https://doi.org/10.1177/1090820X14541956

[14] Gutowski, K.A. (2016) Microfocused Ultrasound for Skin Tightening. Clinics in Plastic Surgery, 43, 577-582. https://doi.org/10.1016/j.cps.2016.03.012

[15] Fabi, S.G. (2015) Noninvasive Skin Tightening: Focus on New Ultrasound Techniques. Clinical, Cosmetic and Investigational Dermatology, 8, 47-52. https://doi.org/10.2147/CCID.S69118

[16] Fabi, S.G. (2014) Microfocused Ultrasound with Visualization for Skin Tightening and Lifting: My Experience and a Review of the Literature. Dermatologic Surgery, 40, S164-S167. https://doi.org/10.1097/DSS.0000000000000233

[17] Vanaman, M., Fabi, S.G. and Cox, S.E. (2016) Neck Rejuvenation Using a Combination Approach: Our Experience and a Review of the Literature. Dermatologic Surgery, 42, S94-S100. https://doi.org/10.1097/DSS.0000000000000699

[18] Chan, N.P., Shek, S.Y., Yu, C.S., Ho, S.G., Yeung, C.K. and Chan, H.H. (2011) Safety Study of Transcutaneous Focused Ultrasound for Non-Invasive Skin Tightening in Asians. Lasers in Surgery and Medicine, 43, 366-375. https://doi.org/10.1002/lsm.21070

[19] Kerscher, M., Nurrisyanti, A.T., Eiben-Nielson, C., Hartmann, S. and Lambert-Baumann, J. (2019) Skin Physiology and Safety of Microfocused Ultrasound with Visualization for Improving Skin Laxity. Clinical, Cosmetic and Investigational Dermatology, 12, 71-79. https://doi.org/10.2147/CCID.S188586

[20] Fabi, S.G., Joseph, J., Sevi, J., Green, J.B. and Peterson, J.D. (2019) Optimizing Patient Outcomes by Customizing Treatment with Microfocused Ultrasound with Visualization: Gold Standard Consensus Guidelines from an Expert Panel. Journal of Drugs in Dermatology, 18, 426-432.

[21] Friedmann, D.P., Bourgeois, G.P., Chan, H.H.L., Zedlitz, A.C. and Butterwick, K.J. (2018) Complications from Microfocused Transcutaneous Ultrasound: Case Series and Review of the Literature. Lasers in Surgery and Medicine, 50, 13-19. https://doi.org/10.1002/lsm.22768

[22] Baumann, L. and Zelickson, B. (2016) Evaluation of Micro-Focused Ultrasound for Lifting and Tightening Neck Laxity. Journal of Drugs in Dermatology, 15, 607-614. 